This item was submitted to Loughborough's Research Repository by the author.

Items in Figshare are protected by copyright, with all rights reserved, unless otherwise indicated.

\title{
On hashing with tweakable ciphers
}

PLEASE CITE THE PUBLISHED VERSION

PUBLISHER

(C) IEEE

VERSION

VoR (Version of Record)

LICENCE

CC BY-NC-ND 4.0

REPOSITORY RECORD

Phan, Raphael C.-W., and Jean-Philippe Aumasson. 2019. "On Hashing with Tweakable Ciphers". figshare. https://hdl.handle.net/2134/5684. 
This item was submitted to Loughborough's Institutional Repository (https://dspace.lboro.ac.uk/) by the author and is made available under the following Creative Commons Licence conditions.

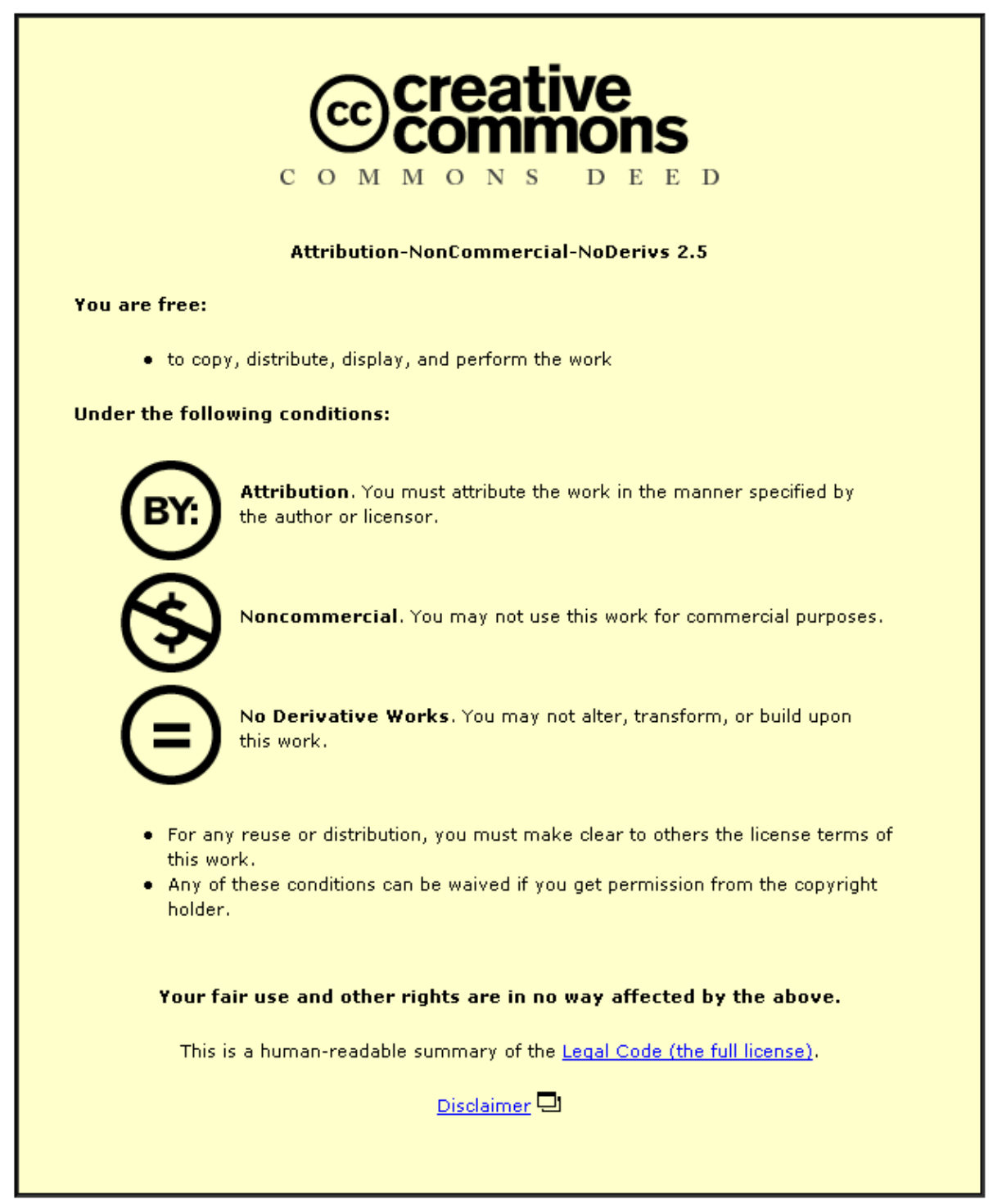

For the full text of this licence, please go to: http://creativecommons.org/licenses/by-nc-nd/2.5/ 


\section{On Hashing With Tweakable Ciphers}

\author{
Raphael C.-W. Phan \\ Loughborough University, UK \\ Email: R.Phan@lboro.ac.uk
}

\author{
Jean-Philippe Aumasson \\ FHNW, Windisch, Switzerland \\ Email: JeanPhilippe.Aumasson@gmail.com
}

\begin{abstract}
Cryptographic hash functions are often built on block ciphers in order to reduce the security analysis of the hash to that of the cipher, and to minimize the hardware size. Well known hash constructs are used in international standards like MD5 and SHA-1. Recently, researchers proposed new modes of operations for hash functions to protect against generic attacks, and it remains open how to base such functions on block ciphers. An attracting and intuitive choice is to combine previous constructions with tweakable block ciphers. We investigate such constructions, and show the surprising result that combining a provably secure mode of operation with a provably secure tweakable cipher does not guarantee the security of the constructed hash function. In fact, simple attacks can be possible when the interaction between secure components leaves some additional "freedom" to an adversary. Our techniques are derived from the principle of slide attacks, which were introduced for attacking block ciphers.
\end{abstract}

\section{INTRODUCTION}

Cryptographic hash functions are key ingredients in numerous schemes like public-key encryption, digital signatures, message-authentication codes, or multiparty functionalities. During the last few years, the focus on hash functions has dramatically increased, because of new dedicated attacks on e.g. MD5 and SHA-1, and new generic attacks-that is, which apply to broad classes of functions. A hash function $h$ should satisfy (at least)

- collision resistance: it should be hard to find distinct inputs $x$ and $x^{\prime}$ such that $h(x)=h\left(x^{\prime}\right)$

- second-preimage resistance: given a random input $x$, it should be hard to find a distinct $x^{\prime}$ such that $h(x)=h\left(x^{\prime}\right)$

- preimage resistance: given $h(x)$ for a random unknown $x$, it should be hard to find a distinct $x^{\prime}$ such that $h(x)=$ $h\left(x^{\prime}\right)$

Critical generic attacks [1]-[3] were presented against the classical Merkle-Damgård (MD) iterative mode of operation, thus threatening all the hash functions using the MD operation mode (for example, MD5 and SHA-1). An MD hash function $H$ hashes a message $M=M_{1} M_{2} \ldots M_{\ell}$ as follows: for $1 \leq i \leq \ell$, compute

$$
h_{i}=f\left(h_{i-1}, M_{i}\right),
$$

where $f$ is called the compression function, and $h_{0}$ is a predefined initialization vector (IV). Finally the function returns the hash value $H(M)=h_{\ell}$.

To prevent from attacks on the MD mode, extended operation modes were proposed (e.g. HAIFA [4], [5]); in this work we focus on Rivest's dithered MD (DMD) mode [6],
[7], for its simplicity and better efficiency. DMD was proposed as a general framework for hash functions, and it remains to be seen how to concretely instantiate the underlying compression function. Furthermore, we know of no concrete hash function construction that employs DMD. The question has been discussed among the community as to whether the upcoming NIST hash function competition [8] should concentrate on only concrete hash function proposals, or split between proposals for operating modes and for compression functions (cf. [9]).

Block cipher-based constructions for hash functions used to build on the MD mode [10], [11] (the so-called $P G V$ schemes) and there is no direct way to extend them to DMD, because of an additional input to the compression function. Recently, ad-hoc efficient constructions were analyzed [12], but it is unclear whether this approach is optimal. A natural approach suggested by Rivest [6], [7] is to use tweakable block ciphers [13] to instantiate DMD hash functions. The model of tweakable block cipher was originally proposed by Liskov, Rivest, and Wagner to define families of permutations for a fixed secret key, thus avoiding the slowdown caused by the key schedule operation.

\section{A. Contribution}

We consider two classes of constructions for DMD hash functions based on tweakable block ciphers, which combine ${ }^{1}$

1) a secure hash mode of operation

2) a secure tweakable block cipher

3) a secure block cipher-based construction

Then, we show that such constructions do not necessarily lead to a secure hash function. More precisely, we apply the idea of slid pairs to find collisions for one of the function classes. Our attacks apply to broad classes of constructions, and are independent of the strength of the underlying block cipher used.

\section{B. Related Work}

Dithering of hash functions appeared with the work of Kelsey and Schneier [1], with generalizations in [4]-[7]. An analysis of dithered hash functions appears in [14], and constructive results were proposed in [12].

Hash functions based on block ciphers recently attracted considerable attention, with several results proving security

\footnotetext{
${ }^{1}$ The notion of security differs for each of these constructions, see the corresponding papers [6], [10], [13] for details.
} 
bounds for constructions with one or more block ciphers [15][18]. Concrete block cipher-based designs include Maelstrom [19] and Grindahl [20], and implicitly the de facto standards MD5, SHA-1, and SHA-2.

The first known application of the sliding techniques [21] [24] to the context of hash functions was by [25]; namely to slide the compression function of SHA-1. This result was extended in [26], and later applied to the SHA based block cipher SHACAL-1. In [27], a slide-style attack was presented on incremental hash functions based on pair block chaining. Recently, Gorski et al. applied sliding to mount distinguishing attacks against Sponge type hash functions [28]; as well as key recovery attacks on Sponge hash function based MACs. The generic hash function attacks by Dean [29], and Kelsey and Schneier [1] exploit a fixed-point of the compression function, which is similar in spirit to slide attacks [21], [22] and to our attacks. Our results are the first known work that slide dithered hash functions.

In [30], attacks were mounted on a hash function mode based on tweakable block ciphers, so called Tweak Chain Hash (TCH). This construction, however, is a conventional hash function (not dithered). The attack on $\mathrm{TCH}$ does not apply to the constructions we consider in this paper.

\section{Definitions}

A block cipher is a map $E:\{0,1\}^{k} \times\{0,1\}^{m} \mapsto\{0,1\}^{m}$, such that $E_{K}(\cdot)=E(K, \cdot)$ is a permutation of $\{0,1\}^{m}$ for all $K \in\{0,1\}^{k}$, and its inverse permutation is written $E^{-1}$. The set of all block ciphers with $k$-bit key and $m$-bit messages is denoted $\operatorname{Bloc}(k, m)$. A block cipher-based hash function is a map $H: \operatorname{Bloc}(k, m) \times D \mapsto R$, where $D \subseteq\{0,1\}^{\star}$ and $R=\{0,1\}^{n}$, defined iteratively by a compression function $f: \operatorname{Bloc}(k, m) \times\{0,1\}^{n_{1}} \times\{0,1\}^{n_{2}} \mapsto\{0,1\}^{n_{2}}$, where $n_{1}$ is the size of a message block, and $n_{2}$ the size of chaining values. In the remainder of the paper, we assume $m=n_{1}=n_{2}=n$.

\section{A. Hash Compression Function Modes based on Block Ci- phers}

Among the 12 provably secure hash compression function modes presented in [10], we will focus on the most popular ones (which are used in all concrete hash designs):

- the Matyas-Meyer-Oseas [31] (MMO) mode, which constructs the compression function by setting

$$
h_{i}=E_{h_{i-1}}\left(M_{i}\right) \oplus M_{i} .
$$

- the Davies-Meyer mode, somehow the dual of MMO, is used in the MD5 and SHA functions:

$$
h_{i}=E_{M_{i}}\left(h_{i-1}\right) \oplus h_{i-1} .
$$

- the Miyaguchi-Preneel mode [32], [33], notably employed in Whirlpool [34], where the underlying block cipher is a variant of Rijndael:

$$
h_{i}=E_{h_{i-1}}\left(M_{i}\right) \oplus h_{i-1} \oplus M_{i} .
$$

\section{B. Tweakable Block Ciphers}

Tweakable block ciphers [13] aim to achieve the "best of both worlds" (security and efficiency) for block cipher-based hashing, since they allow to use an input-dependent permutation, thus avoiding the time-consuming key schedule-the additional input (tweak) being injected in a simplistic fashion.

Formally, a tweakable block cipher has three inputs: a key $K \in\{0,1\}^{k}$, a tweak $T \in\{0,1\}^{t}$ and a message $M \in\{0,1\}^{n}$; it produces a ciphertext $C \in\{0,1\}^{n}$ :

$$
\tilde{E}:\{0,1\}^{k} \times\{0,1\}^{t} \times\{0,1\}^{n} \mapsto\{0,1\}^{n} .
$$

We write $\tilde{E}_{K}(T, M)$ as shorthand for $\tilde{E}(K, T, M)$. In [13], two tweakable block ciphers are constructed from conventional block ciphers:

- TEXE (tweakable cipher formed by two $E$ boxes sandwiching an XOR), which is inspired from CBC-MAC and is defined as

$$
\tilde{E}_{K}(T, M)=E_{K}\left(T \oplus E_{K}(M)\right) .
$$

It was proven [13] that TEXE is a secure tweakable cipher in the sense of indistinguishability from a family of random permutations parametrized by the tweak.

- TFX (inspired from the generalized DESX construction FX of Kilian and Rogaway [35], [36]) defines the scheme

$$
\tilde{E}_{K}(T, M)=E_{K}(M \oplus U(T)) \oplus U(T),
$$

where $U$ is a universal hash function TFX is strongly (chosen-ciphertext) secure in the sense of indistinguishability from a family of random permutations parametrized by the tweak (see [13] for details).

\section{Dithered Hash Functions (DMD)}

Dithering is a generalization of the countermeasure proposed by Kelsey and Schneier [1] to prevent attacks [1], [29] based on message block repetition and fixed-points. This type of iterated hash uses a sequence of dither values $D=d_{1} \ldots d_{\ell}$, which is public and static.

A dithered Merkle-Damgård (DMD) hash function, as defined in [6], [7], takes as input an IV, a message $M=$ $M_{1} M_{2} \ldots M_{\ell}$, a dither sequence $D=d_{1} d_{2} \ldots d_{\ell}$, and produces an output $H_{D}(M)$ as follows: for $1 \leq i \leq \ell$, compute

$$
h_{i}=f\left(h_{i-1}, M_{i}, d_{i}\right)
$$

where $f$ is the compression function, the $h_{i}$ 's are chaining variables; $h_{0}$ is the IV and the dither values $d_{0}, \ldots, d_{\ell-1}$ have a zero most significant bit (MSB), and $d_{\ell}$, the last dither value, has nonzero MSB.

In the above definition, a special MSB encoding of $d_{i}$ differentiates the last block from other blocks. This feature was proposed to avoid a complex message padding rule (unlike classical MD functions, which append to a message the encoding of its bit length). 


\section{DMD CONSTRUCTIONS}

We consider constructions of DMD hash functions where the compression function is instantiated with a tweakable block cipher, in one of the 12 provably secure PGV modes [11]. The dither input $d_{i}$ of the compression function is directed to the tweak input $T$ of the tweakable block cipher as suggested in [6], [7]. We focus on the MMO mode to simplify the description, though our results equally apply to other modes as detailed in Section V-A.

\section{A. DMD-TEXE in MMO Mode}

This construction of a DMD function combines the TEXE and $\mathrm{MMO}$ schemes, which respectively add a new input slot and construct a secure compression function. Following our above definitions, DMD-TEXE with the MMO scheme defines

$$
\begin{aligned}
h_{i} & =f\left(h_{i-1}, M_{i}, d_{i}\right) \\
& =\tilde{E}_{h_{i-1}}\left(d_{i}, M_{i}\right) \oplus M_{i} \\
& =E_{h_{i-1}}\left(d_{i} \oplus E_{h_{i-1}}\left(M_{i}\right)\right) \oplus M_{i} .
\end{aligned}
$$

This construction, however, is inefficient, since each call to the compression function requires two encryptions with the block cipher $E$, plus one key schedule (both encryptions use the same key).

\section{B. DMD-TFX in MMO Mode}

This construction is more efficient than DMD-TEXE, since it requires only one encryption (and the key schedule), plus a call to a universal hash function, which is generally faster than the block cipher in practice. This construction defines:

$$
\begin{aligned}
h_{i} & =f\left(h_{i-1}, M_{i}, d_{i}\right) \\
& =\tilde{E}_{h_{i-1}}\left(d_{i}, M_{i}\right) \oplus M_{i} \\
& =E_{h_{i}-1}\left(M_{i} \oplus U\left(d_{i}\right)\right) \oplus U\left(d_{i}\right) \oplus M_{i} \\
& =E_{h_{i}-1}\left(M_{i} \oplus U\left(d_{i}\right)\right) \oplus\left(M_{i} \oplus U\left(d_{i}\right)\right) .
\end{aligned}
$$

\section{COLlision ATtACK}

We show how to mount a free-start collision attack on DMD-TFX in MMO mode, resulting in a pair of colliding messages $M$ and $M^{\prime}$ for a predefined $I V$ and another $I V^{\prime}$. The attack goes as follows, for an arbitrary dither sequence $D=D=d_{1} \ldots d_{\ell+1}$ :

1) choose an arbitrary $(\ell+1)$-block message $M=$ $M_{1} M_{2} \ldots M_{\ell+1}$, and compute $H_{D}(M)$

2) define a $\ell$-block message $M^{\prime}=M_{1}^{\prime} \ldots M_{\ell}^{\prime}$, where

$$
M_{i}^{\prime}=M_{i+1} \oplus U\left(d_{i+1}\right) \oplus U\left(d_{i}\right) .
$$

3) compute $H_{D}\left(M^{\prime}\right)$ using the IV $h_{0}^{\prime} \neq h_{0}$ defined as

$$
h_{0}^{\prime}=f\left(h_{0}, M_{1}, d_{1}\right)=h_{1} .
$$

Now, Eq. (1) and (2) yield

$$
\begin{aligned}
h_{1}^{\prime} & =f\left(h_{0}^{\prime}, M_{1}^{\prime}, d_{1}\right) \\
& =E_{h_{0}^{\prime}}\left(M_{1}^{\prime} \oplus U\left(d_{1}\right)\right) \oplus\left(M_{1}^{\prime} \oplus U\left(d_{1}\right)\right) \\
& =E_{h_{1}}\left(M_{2} \oplus U\left(d_{2}\right)\right) \oplus\left(M_{2} \oplus U\left(d_{2}\right)\right) \\
& =f\left(h_{1}, M_{2}, d_{2}\right) \\
& =h_{2} .
\end{aligned}
$$

Then, by induction,

$$
\begin{aligned}
h_{i}^{\prime} & =f\left(h_{i-1}^{\prime}, M_{i}^{\prime}, d_{i}\right) \\
& =E_{h_{i-1}^{\prime}}\left(M_{i}^{\prime} \oplus U\left(d_{i}\right)\right) \oplus\left(M_{i}^{\prime} \oplus U\left(d_{i}\right)\right) \\
& =E_{h_{i}}\left(M_{i+1} \oplus U\left(d_{i+1}\right)\right) \oplus\left(M_{i+1} \oplus U\left(d_{i+1}\right)\right) \\
& =f\left(h_{i}, M_{i+1}, d_{i+1}\right) \\
& =h_{i+1} .
\end{aligned}
$$

Eventually we have $h=h_{\ell+1}$ and $h^{\prime}=h_{\ell}^{\prime}$, and thus $H_{D}(M)=H_{D}\left(M^{\prime}\right)$, i.e. a collision.

\section{A. Generalization}

This attack generalizes to other DMD-TFX in PGV modes, for example when the underlying block cipher-based compression function sets

$$
E_{h_{i-1}}\left(M_{i} \oplus h_{i-1}\right) \oplus M_{i} \oplus h_{i-1},
$$

or

$$
E_{h_{i-1}}\left(M_{i} \oplus h_{i-1}\right) \oplus M_{i} .
$$

These are the PGV mode constructions called $f_{2}$ and $f_{4}$ in [11]. Our attack also applies to the PGV mode popularly known as Miyaguchi-Preneel.

More generally, our attack can in general be applied to DMD hash functions with symmetric mixing of the message block and the dither input. Examples of this kind would be inspired from perceptual hash functions in image authentication applications [37], [38] of the field of image processing, from which the notion of "dithering" is inspired. In such hash functions, the dither input is mixed within the compression function in the same way as how a message input is mixed, hence the mixing is symmetric.

We can describe a further generalization, when the compression function $f$ can be expressed as

$$
\begin{aligned}
h_{i} & =f\left(h_{i-1}, M_{i}, d_{i}\right) \\
& =f^{\prime}\left(h_{i-1}, f_{1}\left(M_{i}\right) \otimes f_{2}\left(d_{i}\right)\right)
\end{aligned}
$$

where $\otimes$ and $f_{1}$ are arbitrary invertible functions, and $f_{2}$ is an arbitrary function, not necessarily invertible.

Our attack can be applied to this case too, by choosing $M_{i}^{\prime}$ such that

$$
f_{1}\left(M_{i}^{\prime}\right) \otimes f_{2}\left(d_{i}\right)=f_{1}\left(M_{i+1}\right) \otimes f_{2}\left(d_{i+1}\right),
$$

i.e., we choose:

$$
M_{i}^{\prime}=f_{1}^{-1}\left\{\left[f_{1}\left(M_{i+1}\right) \otimes f_{2}\left(d_{i+1}\right)\right] \otimes^{-1} f_{2}\left(d_{i}\right)\right\} .
$$

Note that $f_{1}$ and $f_{2}$ can be defined to also take the chaining variable $h_{i-1}$ as input, and our attack equally applies.

\section{B. Applicability to DMD-TEXE Functions}

Functions based on the TEXE construction resist our attacks since the mixing between $M_{i}$ and $d_{i}$ is not invertible, with respect to expressing $M_{i}$ from the above equation in terms of the other variables $h_{i}, h_{i-1}$ and $d_{i}$.

It might seem counter-intuitive that DMD is insecure against our attack when instantiated with TFX yet is resistant when 
instantiated with the essentially weaker [13] TEXE. This might be because the difference between the two security notions achieved by TFX and TEXE is in terms of the access to the decryption oracle, which does not appear to be useful since PGV modes only make use of the underlying block cipher in the encryption direction.

\section{CONCLusions}

Attacks such as [1], [29] seem to provide support for the hypothesis [6], [7], [39] that the adversary attacking an MD hash function has too much control over the message block input to the compression function, and so this control should be restricted [1], [6], [7], e.g. with dithering which furthermore makes the process of hashing a message block dependent on its position within the entire message [39], or alternatively makes the compression function round-dependent. DMD hash functions therefore increase the security guarantees, by using different compressions of the message at each iteration. However, care should be taken when using dithers for this restriction, since a dither input, although is predefined, is another input channel and another degree of freedom that an adversary could potentially exploit; as our attacks showed.

Viewed from another perspective, even if two copies of the hash iteration are out of phase thus leading to differences between the copies due to differing round positions and hence different dither inputs, an adversary can still use message block differences to shift the position dependence, and subsequently offset them.

A countermeasure for our attack is to append to the message its bit length; this makes the function less efficient and in fact clashes with the DMD design objective of achieving efficiency and of eliminating the need for conventional message padding. It remains open, though, whether concrete hash functions should be based on block ciphers, or be dedicated designs in order to satisfy the very particular security requirements of a hash function, not necessarily captured by those of block ciphers (cf. notions like indifferentiability, seedincompressibility, secure MAC, etc.).

Biham [4] highlights an important point in the design and analysis of hash functions: that the same technology, principles and design criteria as block ciphers should be used for hash functions. He further explains that this is supported by the fact that the block cipher design criteria of strong avalanche criterion (SAC) and strong diffusion, ensures that neutral bits (used to attack hash functions) cannot exist; and a good example is the case of MDx and SHAx hash functions that have slow diffusion which probably explains why it is easier to control differences through their rounds than it is to do so for block ciphers. Furthermore, it is well known that block ciphers and hash functions can be converted to each other through some suitable mode of operation. To this purpose, we have:

1) considered the security of the dithered MD hash function construction and how its security is affected by its interactions with the underlying tweakable block cipher and corresponding block cipher-based compression function mode.

2) shown how to apply the block cipher cryptanalytic technique of slide attacks to mount collision attacks on hash functions.

For the first point, our results show that caution needs to be exercised when interacting primitives even if they are provably secure: in this case, the secure tweakable cipher TFX and the secure MMO (resp. Miyaguchi-Preneel, $f_{2}, f_{4}$ ) modes.

For the second point, we contrast between a block cipher and a hash function in terms of the difficulty of applying the kind of attacks treated in this paper. In some PGV modes of operation, the round key input $K$ of the block cipher is the message block input $M_{i}$ of the hash function, and while there exists a key schedule in block ciphers, hash functions either have no schedule to process message blocks or the schedule is linear (e.g. SHA). Therefore, round keys going into each round of the block cipher depend on a master key via a complex key schedule, and thus the round keys are difficult to control, whereas the message blocks to the hash function can be easily controlled by qan attacker.

Furthermore, an adversary attacking a block cipher has no control over the intermediate states of the cipher, unless one considers the context of related-key attacks, which even in that case only gives restricted control in the sense that an adversary may be able to differentially affect the round keys of two or more copies of the encryption iteration but yet not know let alone be able to control what the round key values are. In contrast for the case of hash functions, the message blocks that are in some PGV modes the analog of round keys in block ciphers, are not only known but can be chosen by the adversary. All these points indicate that it should be easier to mount sliding techniques to hash functions.

Slide attacks on block ciphers require some form of periodicity for the encryption or decryption iteration, while for hash functions we demonstrated that this is no longer necessary because the difference due to sliding different rounds can be offset by the mixing of message block differences and dither inputs. And while the dithering mechanisms proposed in [6], [7] prevent the attacks in [1], [29] by eliminating message block repetitions, our attacks apply even if there are no repetitions in the sequence.

Our work further supports the view [39] that the role that sequences play in iterated cryptographic constructions needs to be further studied. In this particular case, nonrepetitive sequences would complicate slide attacks for block ciphers but apparently not necessarily for hash functions.

\section{REFERENCES}

[1] J. Kelsey and B. Schneier, "Second preimages on n-bit hash functions for much less than $2^{\mathrm{n}}$ work." in EUROCRYPT, ser. LNCS, R. Cramer, Ed., vol. 3494. Springer, 2005, pp. 474-490.

[2] J. Kelsey and T. Kohno, "Herding hash functions and the Nostradamus attack," First NIST Cryptographic Hash Function Workshop, 2005.

[3] —_ "Herding hash functions and the Nostradamus attack." in EUROCRYPT, ser. LNCS, S. Vaudenay, Ed., vol. 4004. Springer, 2006, pp. 183-200. 
[4] E. Biham, "Recent advances in hash functions - the way to go," 2005, presented at the ECRYPT Hash Function Workshop, 2005.

[5] E. Biham and O. Dunkelman, "A framework for iterative hash functions HAIFA," Cryptology ePrint Archive, Report 2007/278, 2007, previously presented at the second NIST Hash Function Workshop, 2006.

[6] R. L. Rivest, "Abelian square-free dithering for iterated hash functions," ECRYPT Workshop on Hash Functions, 2005.

[7] _ , "Abelian square-free dithering for iterated hash functions," NIST Hash Function Workshop, 2005.

[8] NIST, "Cryptographic hash competition," 2008, http://www.nist.gov/hash-function/.

[9] D. J. Bernstein, NIST's hash mailing list, post on June 8, 2007.

[10] B. Preneel, R. Govaerts, and J. Vandewalle, "Hash functions based on block ciphers: A synthetic approach," in CRYPTO, ser. LNCS, D. R. Stinson, Ed., vol. 773. Springer, 1993, pp. 368-378.

[11] J. Black, P. Rogaway, and T. Shrimpton, "Black-box analysis of the block-cipher-based hash-function constructions from pgv." in CRYPTO, ser. LNCS, M. Yung, Ed., vol. 2442. Springer, 2002, pp. 330-335.

[12] J.-P. Aumasson and R. C.-W. Phan, "How (not) to efficiently dither blockcipher-based hash functions?" in AFRICACRYPT, ser. LNCS, S. Vaudenay, Ed., vol. 5023. Springer, 2008, pp. 308-324.

[13] M. Liskov, R. L. Rivest, and D. Wagner, "Tweakable block ciphers." in CRYPTO, ser. LNCS, M. Yung, Ed., vol. 2442. Springer, 2002, pp. $31-46$.

[14] E. Andreeva, C. Bouillaguet, P.-A. Fouque, J. J. Hoch, J. Kelsey, A. Shamir, and S. Zimmer, "Second preimage attacks on dithered hash functions," in EUROCRYPT, ser. LNCS, N. P. Smart, Ed., vol. 4965. Springer, 2008, pp. 270-288.

[15] M. Stam, "Another glance at blockcipher based hashing," Cryptology ePrint Archive, Report 2008/071, 2008.

[16] T. Shrimpton and M. Stam, "Building a collision-resistant compression function from non-compressing primitives," in ICALP (2), ser. LNCS, L. Aceto, I. Damgård, L. A. Goldberg, M. M. Halldórsson, A. Ingólfsdóttir, and I. Walukiewicz, Eds., vol. 5126. Springer, 2008, pp. 643-654.

[17] P. Rogaway and J. P. Steinberger, "Security/efficiency tradeoffs for permutation-based hashing," in EUROCRYPT, ser. LNCS, N. P. Smart, Ed., vol. 4965. Springer, 2008, pp. 220-236.

[18] - "Constructing cryptographic hash functions from fixed-key blockciphers," in CRYPTO, ser. LNCS, D. Wagner, Ed., vol. 5157. Springer, 2008, pp. 433-450.

[19] D. G. Filho, P. Barreto, and V. Rijmen, "The Maelstrom-0 hash function," in 6th Brazilian Symposium on Information and Computer Security, 2006.

[20] L. R. Knudsen, C. Rechberger, and S. S. Thomsen, "The Grindahl hash functions," in FSE, ser. LNCS, A. Biryukov, Ed., vol. 4593. Springer, 2007, pp. 39-57.

[21] A. Biryukov and D. Wagner, "Slide attacks." in FSE, ser. LNCS, L. R. Knudsen, Ed., vol. 1636. Springer, 1999, pp. 245-259.

[22] —-, "Advanced slide attacks." in EUROCRYPT, ser. LNCS, B. Preneel, Ed., vol. 1807. Springer, 2000, pp. 589-606.

[23] R. C.-W. Phan and S. Furuya, "Sliding properties of the des key schedule and potential extensions to the slide attacks," in ICISC, ser. LNCS, P. J. Lee and C. H. Lim, Eds., vol. 2587. Springer, 2002, pp. 138-148.

[24] R. C.-W. Phan, "Advanced slide attacks revisited: Realigning slide on des," in Mycrypt, ser. LNCS, E. Dawson and S. Vaudenay, Eds., vol. 3715. Springer, 2005, pp. 263-276.

[25] D. Wagner, "A slide attack on SHA-1," Unpublished manuscript, June 2001.

[26] M.-J. O. Saarinen, "Cryptanalysis of block ciphers based on SHA-1 and MD5." in FSE, ser. LNCS, T. Johansson, Ed., vol. 2887. Springer, 2003, pp. 36-44.

[27] R. C.-W. Phan and D. Wagner, "Security considerations for incremental hash functions based on pair block chaining," Computers and Security, vol. 25, no. 2, pp. 131-136, 2006.

[28] M. Gorski, S. Lucks, and T. Peyrin, "Slide attacks on a class of hash functions," in ASIACRYPT, ser. LNCS, J. Pieprzyk, Ed., vol. 5350. Springer, 2008, pp. 143-160.

[29] R. D. Dean, "Formal aspects of mobile code security." Ph.D. dissertation, Princeton University, 1999.

[30] J. Black, M. Cochran, and T. Shrimpton, "On the impossibility of highlyefficient blockcipher-based hash functions." in EUROCRYPT, ser. LNCS, R. Cramer, Ed., vol. 3494. Springer, 2005, pp. 526-541.
[31] S. Matyas, C. Meyer, and J. Oseas, "Generating strong one-way functions with cryptographic algorithm," IBM Technical Disclosure Bulletin, vol. 27, no. 10A, pp. 5658-5659, 1985.

[32] S. Miyaguchi, K. Ohta, and M. Iwata, "128-bit hash function (N-Hash)." NTT Review, vol. 2, pp. 128-132, 1990.

[33] B. Preneel, "Analysis and design of cryptographic hash functions." Ph.D. dissertation, Katholieke Universiteit Leuven, January 1993.

[34] P. Barreto and V. Rijmen, "The Whirlpool hashing function," First Open NESSIE Workshop, 2000.

[35] J. Kilian and P. Rogaway, "How to protect DES against exhaustive key search." in CRYPTO, ser. LNCS, N. Koblitz, Ed., vol. 1109. S Springer, 1996, pp. 252-267.

[36] — "How to protect DES against exhaustive key search (an analysis of DESX)." Journal of Cryptology, vol. 14, no. 1, pp. 17-35, 2001.

[37] M. Johnson and K. Ramchandran, "Dither-based secure image hashing using distributed coding," in ICIP, vol. 2. IEEE, 2003, pp. 751-754.

[38] A. Swaminathan, Y. Mao, and M. Wu, "Robust and secure image hashing," IEEE Transactions on Information Forensics and Security, vol. 1, pp. 215-230, 2006.

[39] C. Bouillaguet, P.-A. Fouque, A. Shamir, and S. Zimmer, "Second preimage attacks on dithered hash functions," Cryptology ePrint Archive, Report 2007/395, 2007.

[40] R. Cramer, Ed., Advances in Cryptology - EUROCRYPT 2005, 24th Annual International Conference on the Theory and Applications of Cryptographic Techniques, Aarhus, Denmark, May 22-26, 2005, Proceedings, ser. LNCS, vol. $3494 . \quad$ Springer, 2005.

[41] M. Yung, Ed., Advances in Cryptology - CRYPTO 2002, 22nd Annual International Cryptology Conference, Santa Barbara, California, USA, August 18-22, 2002, Proceedings, ser. LNCS, vol. 2442. Springer, 2002.

[42] N. P. Smart, Ed., Advances in Cryptology - EUROCRYPT 2008, 27th Annual International Conference on the Theory and Applications of Cryptographic Techniques, Istanbul, Turkey, April 13-17, 2008. Proceedings, ser. LNCS, vol. 4965. Springer, 2008. 